\title{
AN ANTI-COLLISION METHOD OF SLIP BARREL FOR AUTOMATIC SHIP LOADING IN BULK TERMINAL
}

\author{
SHEN Yang \\ Higher Technical College, Shanghai Maritime University, Shanghai, China
}

\begin{abstract}
During the loading operation of a ship loader in bulk terminal, in order to bring bulk cargo, such as coal and ore, precisely into every corner of the cabin and not to raise dust at the same time, the slip barrel mechanism usually is placed fully inside the ship cabin near the cargo as close as possible. However, if too close, it will increase the risk of collision between slip barrel and cargo, which poses a security risk to loading operation.

This article proposes an anti-collision method for slip barrel in the process of automatic ship loading in bulk terminal. This method can accurately position the cargo of cabin which is blocked by slip barrel and shovel, and provide a reliable guarantee for the automatic loading operation. Firstly, this method use an array of laser radar mounted on the maintenance platforms to perceive the collision objects around the slip barrel, including the cargo and cabin. Meanwhile, it provides a compensation algorithm to reduce the effect of anti-collision recognition brought by dust and various obstacles during the operation. According to the calculated effective distance between the cargo and slip barrel, the automatic control system of ship loading can adjust the loading operation on a real-time basis. In the end, this article analyze and illustrate the reliability and validity of this method by the case of the application in automatic ship loading system in Tianjin coal terminal.
\end{abstract}

Keywords: Automatic Ship Loading, Bulk Terminal, Anti-Collision, Slip Barrel

\section{INTRODUCTION}

In recent years, with the development of information technology and automation equipment or technology, they are widely used in the field of ports. A number of automated and intelligent terminals have been built in succession in the world [1][2]. In the next few decades, it is foreseeable that in order to improve their competitiveness in the related business, automation will not only be preferred at the planning stage for new terminals, but for the existing terminals [3]. These terminal will complete the automatic transformation of the machinery equipment to meet today's increasingly high labor costs and increasingly complex loading and unloading requirements [4].

Among the existing automated terminals, container terminals account for a large majority while the number of automated bulk terminals are relatively small [5][6][7]. This is mainly due to the continuity of the cargo transport, the randomness of the form of cargo and the poor operating environment. These factors have brought serious challenges to the automatic operation of bulk terminals. Regardless of the shape of cargo perception, the precise control of realtime decision-making and the equipment, automated bulk terminals are made to extremely high requirements [8].

As the main working machine of the bulk terminal, the automation degree of the ship loader directly represents the automatic level of the whole terminal. When operating, the factors, such as the structure of the ship loader, the shape of bulk ship, the orientation of the hatch cover, the size of the 
cabin, the type and form of the cargo, dust and pitch angle, will affect the safety and efficiency of the automatic loading operation [9][10][11]. But among all these factors, the real direct one is slip barrel mechanism. So How to reduce the possibility of collision with ship or cargo is the point of research. In some previous studies, some anti-collision protection methods during the period of the port machinery operation have been proposed. Mi Chao et al. has devised an algorithm for ship identification in the automated process of loading operation which not only can be used to detect the edge of the cabin in order to achieve partly anti-collision effects, but also can automatically generate slip barrel position of the ship loading process based on the size of the cabin [12]. Gang Zhou introduced a method of Distance and velocity measurements in automobile anti-collision application [13]. This method use millimeter-wave radar to realize automobile anti-collision in highway. Frederic Maire proposed an anticollision system based on vision [14]. The system can solve the specific problems of the reflectance of rails and allow the creation of a completely self-calibrating system

In the above studies, how to position the cargo of the cabin is not directly involved. And some research methods are not suitable for the identification of the cargo in the bulk terminals, especially those in the harbor bulk terminals. As for the large ship loader, the shovel connecting with the slip barrel and the thrown cargo occlude various detection sensors installed around the slip barrel. And then the sensors will fail to identify the heap area just below slip barrel, so that the scanning shape of the cargo is not complete, because the top of it is missing.

Consequently, this article proposes an anti-collision method for slip barrel in the process of automatic loading in bulk terminal. This method gets the point cloud data of the cargo in the cabin with laser radar arrays and reconstructs the missing surface contour because of the shielding caused by the throwing shovel and the thrown material. Then it restores the complete cargo contour by means of the compensation algorithm, so that it can position and identify the height of the cargo. The automatic control system of the ship loader can adjust the safe distance between cargo and slip barrel in order to operate safely[15].

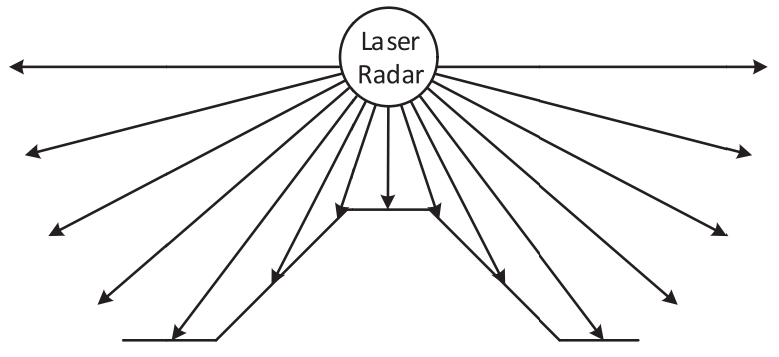

Fig.1 Diagram of LMS scanning

\section{PROBLEM DESCRIPTION}

\section{LASER MEASUREMENT SYSTEM}

LMS is a laser-based multi-angle distance scanner. As shown in Figure 1, the LMS can send and receive the laser in each 0.25 degree. An optical monitor section is made by those lasers. In the optical monitoring section, any target that the laser cannot pass through will be detected. The distance and the sectional plane shape of the target can also be computed by LMS .

As shown in Figure 2, LMSs are installed on the maintenance platform connected the barrel of the ship loader. Those LMSs are grouped a scanning array which can scan the cargo below them at any direction. Because of the shovel and the tossing bulk cargo, the LMS array can only detect the cargo that is not shaded. Figure 3 is the sectional schematic diagram of LMS scanning cargo. In this figure, the center vertical line is center line of slip barrel and the horizontal line above bulk cargo is shovel.

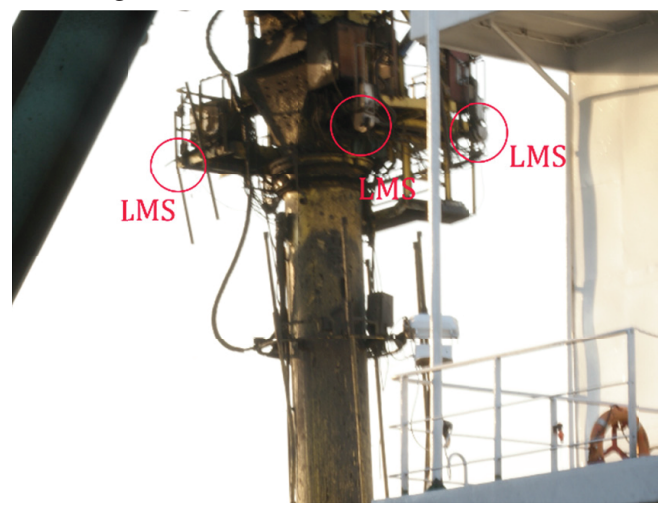

Fig.2 LMSs installed on the ship loader in Tianjin Port

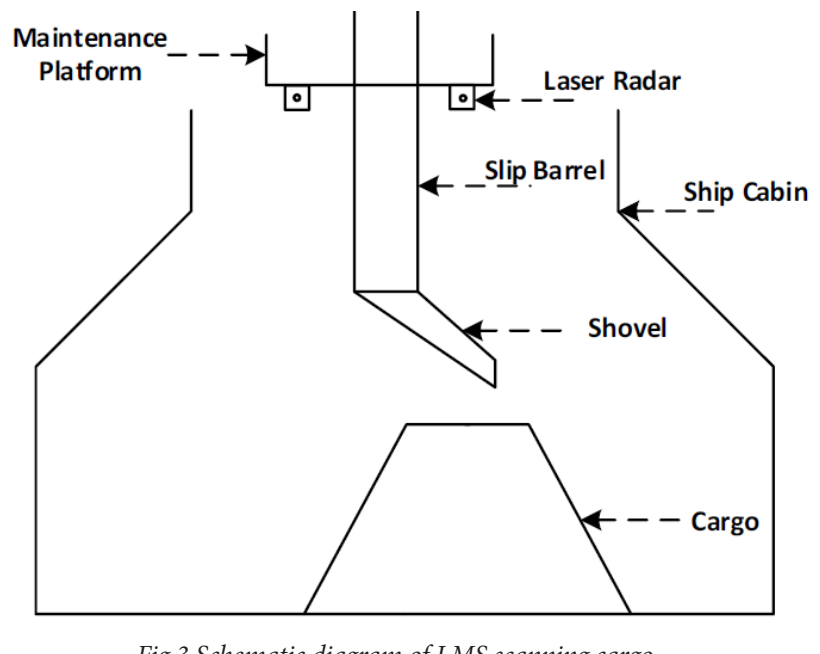

Fig.3 Schematic diagram of LMS scanning cargo

\section{PROBLEM DESCRIPTION}

As shown in Figure 4, the edges of the hold, the cargo inside hold and the shovel are all scanned by the LMS. It is 
obvious that the bulk cargo surface curve is broken for the shovel blocks the laser beam from the LMS. If there is no anything that blocks the laser beam, the curve of the cargo surface should be unbroken. The bulk cargo is tossed from the shovel into the hold, so the point of falling bulk cargo is shaded by the shovel. The point of the falling bulk cargo is also the highest point of the cargo inside the hold which is the key point for the automatic ship loader. This paper mainly discusses how to rebuild the broken cargo surface curve and compute the highest point of the cargo.

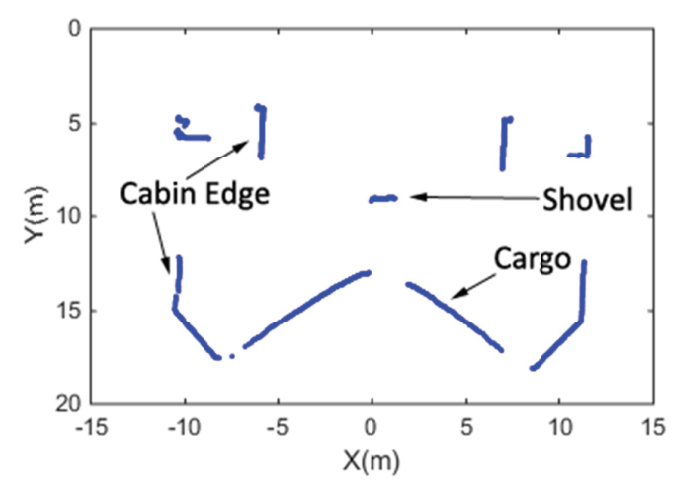

Fig. 4 Scanning result of the LMS

\section{APPROACH FOR BULK CARGO HEIGHT DETECTION}

\section{IMAGE PREPROCESSING}

In the sectional plane generated by LMSs shown in Figure 4 , an image preprocess should be used to remove the edges of the cargo hold. Reference 16 shows a method to inspect the edges of the cargo hold. Then, as shown in Figure 5, the edges in the original image are removed. Only the cargo and shovel information is kept.

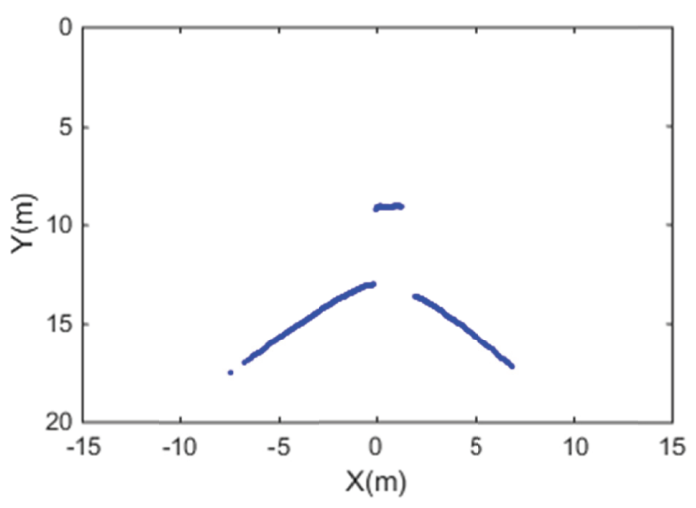

Fig.5 Scanning result after preprocessing

After removing the point cloud of edges of the cargo hold, the point cloud of image should be rasterized for increasing the efficiency of project algorithm and ensuring fast calculation in the automation controller of the ship loader $[17,18]$. Therefore, the set of point cloud would be Rasterized.

$$
\begin{aligned}
& \mathrm{P}_{\text {grid }}(X, Y) \\
& =\left\{\begin{array}{l}
1\left(\exists \mathrm{P}_{\text {cloud }}(x, y), \text { st. } x / D \in[X, X+1), y / D \in[Y, Y+1)\right) \\
0\left(\nexists \mathrm{P}_{\text {cloud }}(x, y), \text { st. } x / D \in[X, X+1), y / D \in[Y, Y+1)\right)
\end{array}\right.
\end{aligned}
$$

Rasterizing process is shown in Equation 1. Assumed that set $\mathrm{P}_{\text {cloud }}(x, y)$ represents the original point cloud edge after removing the hold, where $\mathrm{x}, \mathrm{y}$ denote the horizontal coordinate and vertical coordinate in Cartesian coordinate. The set $\mathrm{P}_{\text {grid }}(X, Y)$ is the results after set $\mathrm{P}_{\text {cloud }}(x, y)$ is rasterized, where $\mathrm{X}, \mathrm{Y}$ represents horizontal coordinate and vertical coordinate in the binary image. $D$ represent the length and width of each grid, the smaller grid size is, the more accurate the project algorithm is, but the greater calculate complex is. When a grid exist one or more points $\mathrm{P}_{\text {cloud }}(x, y)$, the horizontal and vertical coordinates of $\mathrm{P}_{\text {cloud }}(x, y)$ is in the interval $[X, X+1)$ and $[Y, Y+1)$, the grid can be assigned to the value ' 1 '. According to the analysis of LMS parameters and ship hold size, $0.1 \mathrm{~m}$ is a suitable value of D. Image rasterization is shown in Figure 6.

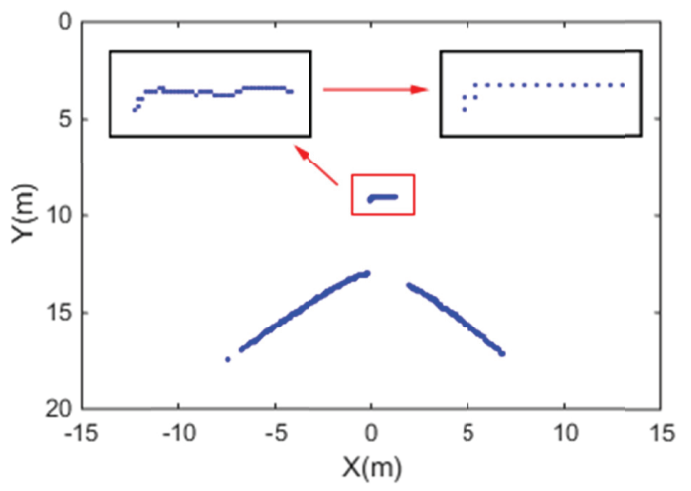

Fig.6 Image rasterization

\section{SHOVEL RECOGNITION}

According to the mechanical relationship between the LMS array and the shovel, the shovel sectional curve in the scanning result should be a short horizontal line. And the shovel curve is always higher than the cargo inside the hold, so there should be a chasm between the shovel curve and the cargo sectional curve. Based on the two distinguishing features, the shovel curve can be separated from the preprocessing image.

The shovel sectional curve is a short horizontal line while the cargo sectional curve is a short oblique line. Therefore, a project algorithm is used to locate the shovel position in the image.

$$
\left\{\begin{array}{c}
C\left(Y_{1}\right)=\operatorname{Count}\left(\left\{\mathrm{P}_{\text {grid }}\left(X, Y_{1}\right) \mid \mathrm{P}_{\text {grid }}\left(X, Y_{1}\right)=1\right\}\right) \\
\vdots \\
C\left(Y_{n-1}\right)=\operatorname{Count}\left(\left\{\mathrm{P}_{\text {grid }}\left(X, Y_{n-1}\right) \mid \mathrm{P}_{\text {grid }}\left(X, Y_{n-1}\right)=1\right\}\right) \\
C\left(Y_{n}\right)=\operatorname{Count}\left(\left\{\mathrm{P}_{\text {grid }}\left(X, Y_{n}\right) \mid \mathrm{P}_{\text {grid }}\left(X, Y_{n}\right)=1\right\}\right) \\
Y_{n}-Y_{n-1}=1
\end{array}\right.
$$


Each grid whose value is 1 in image $\mathrm{P}_{\text {grid }}(X, Y)$ is projected onto the $y$-axis, and counts the number of grid of each line. As shown in Equation 2, $Y_{n}$ represents the vertical coordinate where grid is $\mathrm{n}$ while symbol $\mathrm{C}\left(Y_{n}\right)$ represents the statistical value of grid's count in vertical coordinate $Y_{n}$.

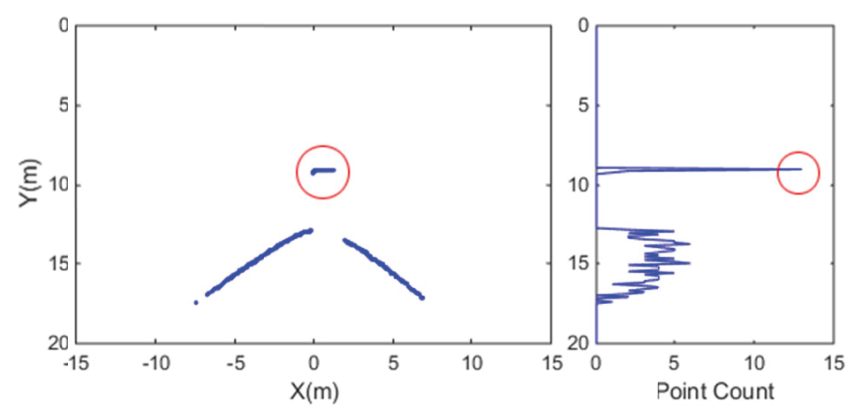

Fig.7 Y-axis projection

As shown in Figure 7, the left image is original binary image coordinate system, the right one is projected coordinate system. In the projected coordinate system, the shovel $y$-axis interval is different from the cargo y-axis interval and they are discontinuous (if they are continuous, that means the shovel location and cargo location is very close. This situation is not allowed in the process of normal operations because of safety). Therefore, it can be concluded that shovel location in a projected coordinate system is in the first continuous interval $R_{1}$.

Assumed that an interval $R=\left[Y_{\text {begin }}, Y_{\text {end }}\right]$ exits in $y$-axis of a projected coordinate system, it must satisfy Equation 3 , all statistical value within the interval is greater than or equal to the threshold $\mathrm{T}$, and the statistical value of the $\mathrm{y}$-axis coordinates $Y_{\text {begin }}-1$ or $Y_{\text {end }}+1$ is less than the threshold T. If the interval $R$ satisfies Equation 3, it can be defined as a continuous interval in y-axis of a projected coordinate system. Through analyzing a large number of experimental data, the value of threshold $\mathrm{T}$ is set to 1 .

$$
\left\{\begin{array}{c}
C\left(Y \in\left[Y_{\text {begin }}, Y_{\text {end }}\right]\right) \geq T \\
C\left(Y=Y_{\text {begin }}-1 \text { or } Y_{\text {end }}+1\right)<T
\end{array}\right.
$$

After finding out the first continuous interval $R_{1}$, we need to find vertical coordinate of the most obvious shovel characteristics where there is the maximum statistical value. As shown in Figure 7, the place of the most obvious shovel characteristics is marked by a red circle. Therefore, by Equation 4, vertical coordinate $Y_{\text {shovel }}$ of shovel can easily be got in a projected coordinate system .

$$
C\left(Y_{\text {shovel }}\right)=\max _{Y \in R_{1}} C(Y)
$$

After obtaining vertical coordinate of shovel in a projected coordinate system, we need to remove all feature points which belong to shovel in the binary image for preventing bad impact on cargo height detection. Searching connected domain from binary image is a good way to achieve the effect of removing shovel $[19,20]$. It uses the connectivity of pixel to quickly find all connected domains from an image and make different markers to different connected domains. There are a larger distance between the shovel curve and cargo curve, so they belong to different connected domains.

In this paper, 8-Connected Neighborhoods is used to define neighborhood range $\mathrm{N}_{P(X, Y)}$ of pixel $P(X, Y)$, the surrounding eight pixels are adjacent to the $P(X, Y)$, so the neighborhood of $P(X, Y)$ need to satisfy Equation 5. By Equation 5, a connected domain set $D s$ can be obtained from binary image. And then a point $P\left(X, Y_{\text {shovel }}\right)$ whose y-axis coordinate is equal to $Y_{\text {shovel }}$ can be chosen randomly. The connected domain where the point stay is the one of shovel $D_{\text {shovel }}$. The connected domain can be expressed by Equation 6.

$$
\begin{aligned}
& \mathrm{N}_{P(X, Y)}=\{P(X+i, Y+j) \mid-1 \leq i \leq 1,-1 \leq j \\
& \leq 1, i, j \text { As integer and Not all zero } \\
& D_{\text {shovel }}=\left\{D s \mid P\left(X, Y_{\text {shovel }}\right) \in D s\right\}
\end{aligned}
$$

As shown in Figure 8, several connected domain can be got by searching binary image. And by using a point $P\left(X, Y_{\text {shovel }}\right)$, shovel connected domain can be reversed to find out and easily be removed. Since the dust and spray in loading operation process and the impact of some foggy weather, cargo sectional curves are not necessarily only two connected domain (the left half and the right half). In figure 8 , the different colors mark is defined as different connected domain, the left half cargo sectional curve is divided into three connected domain, but this does not affect the shovel identification.

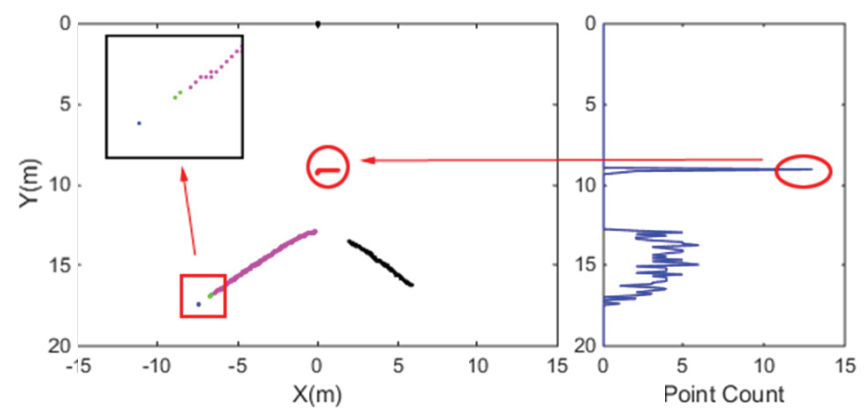

Fig. 8 the process of finding connected domain of shovel

After detecting the connected domain of shovel from image, connected domain of cargo can be remained by Equation 7 and the result is shown in figure 9.

$$
D_{\text {cargo }}=D s-D_{\text {shovel }}
$$




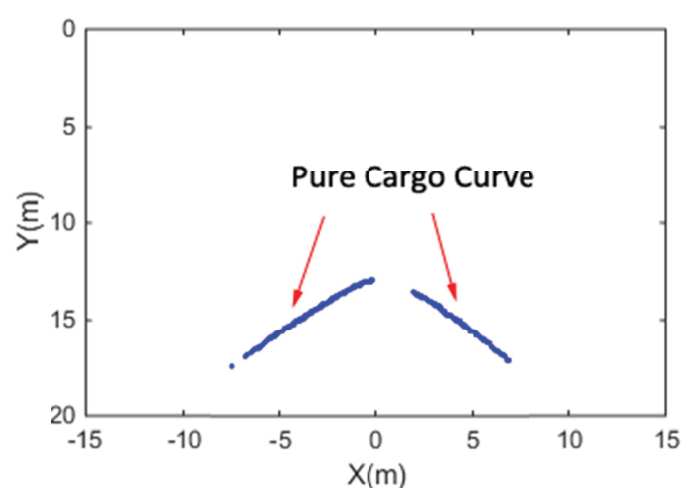

Fig.9 The process of removing connected domain of shovel

\section{CARGO HEIGHT DETECTION}

After removing the section curve of shovel, a pure section curve of cargo is remained. Normally, the location of maximum height of the cargo is the location which is nearest from LMS in vertical direction. However, due to the point cloud of this location is substantially obscured by shovel, so we need to fit the cargo sectional curve which is not obscured, and finally calculate the position of cargo vertex, which is the maximum height of the cargo.

There is many kinds of curve fitting algorithm, such as least square procedure, polynomial fitting, Gaussian function fitting and so on $[21,22]$. The project algorithm in this paper fits the cargo curve by Hough transform. The reason is that Hough transform can meet automatic ship loader's real-time requirements in process of automatic loading operations and increase the efficiency of its implementation in the computer, using the Hough transform to fit the windrow curve, this algorithm is not susceptible to interference noise in the image. At the same time, considering the surface tension and internal friction interaction of the bulk cargo, incline shape of cargo generally approximates a straight line, so the straight line fitting of Hough transform is used.

In the theory of line fitting of Hough transform, each pixel of binary image can be represented by in with polar coordinates of the Equation 8 [23-25]. $\theta$ is the angle between point and the x-axis and $r$ is the distance between point with coordinate origin. As shown in Figure 10, assuming that there is point cloud set $\left\{P\left(x_{1}, y_{1}\right), \cdots, P\left(x_{n-1}, y_{n-1}\right), P\left(x_{n}, y_{n}\right)\right\}$ in the binary image space and $\theta$ and $r$ is defined as the Hough space variables, every point are able to be expressed as a sine curve in the Hough space. These points cloud can be represented by Equations 9. The Hough space is divided to many small grid. By taking the quantification value of $\theta$ into each expression in Equations 9, $r$ can be obtained and mapped into corresponding grid to make counter of the grid plus 1. According to do statistics of each grid counter value, $\theta$ and $r$ of larger value could be as polar parameter of fitted straight line.

In order to ensure project algorithm accuracy and realtime performance, the resolution of $r$ is set to 1 while the resolution of $\theta$ is set to 1 in this algorithm.

$$
\begin{gathered}
r=\cos \theta \times x+\sin \theta \times y \\
\left\{\begin{array}{c}
r=\cos \theta \times x_{1}+\sin \theta \times y_{1} \\
\vdots \\
r=\cos \theta \times x_{n-1}+\sin \theta \times y_{n-1} \\
r=\cos \theta \times x_{n}+\sin \theta \times y_{n}
\end{array}\right.
\end{gathered}
$$

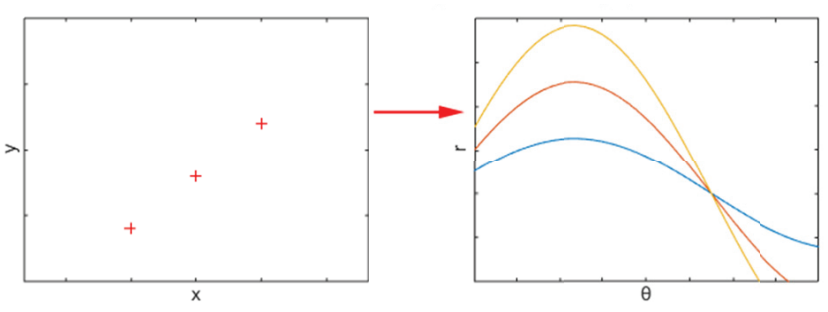

Fig.10 Transition from binary image space to Hough space

As shown in Figure 11, two red straight line $L 1$ and $L 2$ can be obtained by using Hough transform algorithm to fit the cargo incline curve. The four points $P(X 1, Y 1), P(X 2, Y 2)$, $P(X 3, Y 3), P(X 4, Y 4)$ are defined as four endpoints of the two red straight line. The two red straight line can be represented by Equation 10 ..

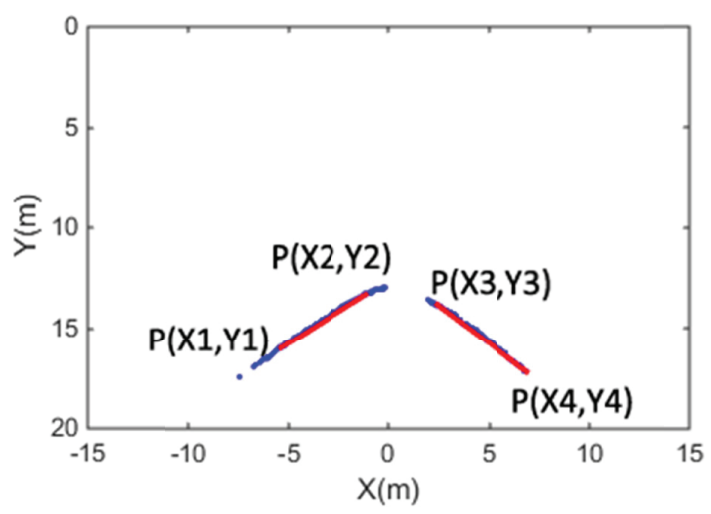

Fig.11 Match cargo incline curve by using Hough transform

$\{(Y 1-Y 2) \times X+(X 2-X 1) \times Y+(X 1 \times Y 2-X 2 \times Y 1)=0$ $\{(Y 3-Y 4) \times X+(X 4-X 3) \times Y+(X 3 \times Y 4-X 4 \times Y 3)=0$

After fitting straight line of the cargo incline, an intersection $P\left(X_{\text {peak }}, Y_{\text {peak }}\right)$ can be obtained by extending the two lines, the coordinate of the intersection is the coordinate of cargo vertex. The $P\left(X_{\text {peak }}, Y_{\text {peak }}\right)$ can be obtained by Equation 11.

$$
P\left(X_{\text {peak }}, Y_{\text {peak }}\right)=\mathrm{L} 1 \cap \mathrm{L} 2
$$


As shown in Figure 12, $Y_{\text {peak }}$ in the point $P\left(X_{\text {peak }}, Y_{\text {peak }}\right)$ is defined as the vertical distance between LMS and bulk cargo vertex position. It does not directly represent the height of the cargo $H_{\text {peak }}$ from the hold bottom surface. As shown in Figure 13, the bottom surface of the hold is substantially perpendicular to the mid perpendicular of LMS, and is farthest in the $y$-axis direction from each other. Therefore, the vertical distance $Y_{\text {bottom }}$ between LMS and hold bottom surface can be quickly determined by Equation 12, which is equal to the largest point cloud $\mathrm{y}$-axis coordinate $Y_{\max }$. Finally, cargo height $H_{\text {peak }}$ can be obtained by Equation 13, which is equal to the difference between the hold bottom vertical coordinate $Y_{\text {bottom }}$ and cargo vertex vertical coordinate $Y_{\text {peak }}$.

$$
\begin{gathered}
Y_{\text {bottom }}=Y_{\max } \\
H_{\text {peak }}=Y_{\text {bottom }}-Y_{\text {peak }}
\end{gathered}
$$

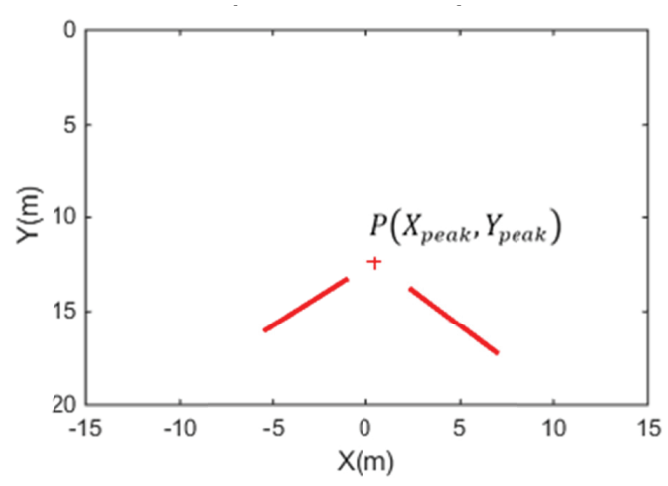

Fig.12 The calculation of cargo height in $y$-axis direction

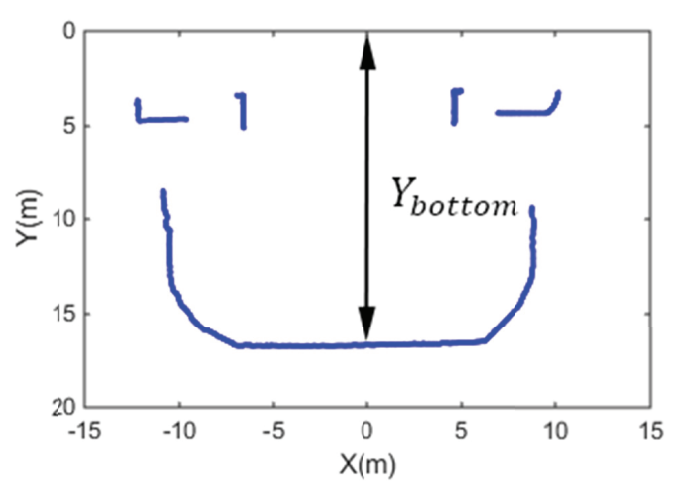

Fig.13 The calculation of ship hold bottom in y-axis direction

\section{EXPERIMENTAL RESULTS}

The method as part of the ship loader automation control system has been applied automatic loading machine in Tianjin Coal Terminal. The automated control system includes a twodimensional laser radar sensor installed in the maintenance platform of slip barrel, rotary motor and servo system, master control existing system in computer of the electrical room and the real-time loading status monitoring system and other subsystems, these sub system communicates with each other by fiber-optic network for data interaction, data acquisition, data processing, active control and status monitoring and a series of tasks of ship loader. With a large number of dock loading business, making slip barrel anti-collision method can be verified by many continuous uninterrupted testing on the basis of the bulk cargo ships of different size and structure.

Two sets of experimental data are selected in all experiments to analyze and explain the anti-collision method of this paper. As shown in Table 1, there is a bulk cargo ship A with five cabins and bulk cargo ship B with four cabins. In this Table, four laser radar are used to scan cargo ship cabin, point cloud data acquisition and calculate error rate of cargo height detection simultaneously in each experiment. The point cloud data acquisition time for each laser radar are selected when a loading operation is nearly completion from starting loading in the cabin. It is about 40 minutes after starting loading. This is because that the distance between cargo and slip barrel is closed at the moment, it is easy to generate collisions between them. In Table 1, the actual value of each radar measurements is taken from the measurements result when the direction of shovel is rotated to other angle where there is no block between the laser radar and cargo directly measured, and the measurement value can be obtained by compensation algorithm when laser radar is blocked. It can be seen by cargo curve compensation and identification algorithm, the error rate of cargo height detection is maintained at about $3 \%$ to $6 \%$, the range is mainly affected by the different laser radar installation location and installation angle of deviation. But the average error rate remained at around $4.5 \%$. The value is considered accurate for a harsh operating environment in bulk cargo terminal. By reasonable threshold adjustments of automatic ship loader control system, the anti-collision subsystem policy can be maintained at a relatively safe level.

Tab.1 two sets of measurement result of LMSs

\begin{tabular}{|l|l|c|c|c|c|}
\hline & & $\begin{array}{c}\text { LMS } \\
\text { No.1 }\end{array}$ & $\begin{array}{c}\text { LMS } \\
\text { No.2 }\end{array}$ & $\begin{array}{c}\text { LMS } \\
\text { No.3 }\end{array}$ & $\begin{array}{c}\text { LMS } \\
\text { Nhip A }\end{array}$ \\
\hline Cabin No.1 & Actual Value & 12.3 & 12.6 & 12.1 & 12.3 \\
\hline & Measurement & 12.7 & 12.0 & 11.4 & 12.8 \\
\hline & Error Rate & $3.3 \%$ & $4.8 \%$ & $5.8 \%$ & $4.1 \%$ \\
\hline Cabin No.2 & Actual Value & 11.3 & 11.5 & 11.1 & 10.9 \\
\hline & Measurement & 11.8 & 12.1 & 10.5 & 11.3 \\
\hline Cabin No.3 & Error Rate & $4.4 \%$ & $5.2 \%$ & $5.4 \%$ & $3.7 \%$ \\
\hline & Measurement & 10.0 & 9.7 & 11.3 & 9.8 \\
\hline & Error Rate & $3.8 \%$ & $4.9 \%$ & $4.6 \%$ & $3.9 \%$ \\
\hline Cabin No.4 & Actual Value & 11.2 & 12.0 & 11.8 & 11.5 \\
\hline & Measurement & 10.8 & 12.6 & 12.4 & 11.9 \\
\hline & Error Rate & $3.6 \%$ & $5.0 \%$ & $5.1 \%$ & $3.5 \%$ \\
\hline Cabin No.5 & Actual Value & 12.1 & 12.4 & 12.3 & 12.9 \\
\hline & Measurement & 12.6 & 13.0 & 11.7 & 12.3 \\
\hline & & & & & 10.2 \\
\hline
\end{tabular}




\begin{tabular}{|l|l|c|c|c|c|}
\hline & Error Rate & $4.1 \%$ & $4.8 \%$ & $4.9 \%$ & $4.7 \%$ \\
\hline Ship B & & & & & \\
\hline Cabin No.1 & Actual Value & 10.8 & 10.5 & 10.8 & 10.3 \\
\hline & Measurement & 11.2 & 11.0 & 10.2 & 10.7 \\
\hline & Error Rate & $3.7 \%$ & $4.8 \%$ & $5.6 \%$ & $3.9 \%$ \\
\hline Cabin No.2 & Actual Value & 11.6 & 11.7 & 12.2 & 11.9 \\
\hline & Measurement & 11.2 & 12.2 & 12.8 & 12.3 \\
\hline & Error Rate & $3.4 \%$ & $4.3 \%$ & $4.9 \%$ & $3.4 \%$ \\
\hline Cabin No.3 & Actual Value & 11.1 & 11.5 & 10.5 & 11.4 \\
\hline & Measurement & 10.7 & 11.0 & 10.0 & 11.0 \\
\hline & Error Rate & $3.6 \%$ & $4.3 \%$ & $4.8 \%$ & $3.5 \%$ \\
\hline Cabin No.4 & Actual Value & 11.7 & 12.3 & 12.1 & 11.6 \\
\hline & Measurement & 12.1 & 11.8 & 11.4 & 11.1 \\
\hline & Error Rate & $3.4 \%$ & $4.1 \%$ & $5.8 \%$ & $4.3 \%$ \\
\hline
\end{tabular}

safe distance between cargo and slip barrel. It can reduce the impact accidents and fatigues for a long time of operations monitor personnel and realize safe, efficient operation in ship loading.

\section{REFERENCES}

1. Nadilo Branko, "BULK CARGO TERMINAL IN PLOCE PORT International cooperation on the project," GRADEVINAR, Vol. 66, No. 10, pp. 953-964 (2014)

2. Bugaric, U.; Petrovic, D., "Increasing the capacity of terminal for bulk cargo unloading," SIMULATION MODELLING PRACTICE AND THEORY, Vol. 15 No. 10 pp. 1366-1381 (2007)

The error rate of cargo height detection against Ship A and Ship B were recorded in four time node of operation. When around 10 minutes at the start of the operation, the error rate is higher, about $6 \%$, since the cargo capacity in the cabin is small, the distance between cargo and laser radar is greater, more dust could be generated by the material falling. However, with the extension of loading time, cabin cargo increased gradually and the distance between cargo and laser radar is reduced, the recognition accuracy is increasing and the error rate is stabilized at $4 \%$. At the same time, it can be found that the error rate of the Ship B when 10 minutes after loading is smaller than Ship A. The comparison indicate that recognition accuracy of small ship is better than the big one due to the influence of size and depth of the cabin.

Tab.2 Error rate of cargo height in four time node of operation

\begin{tabular}{|l|c|c|c|c|}
\hline & $10 \mathrm{~min}$ & $20 \mathrm{~min}$ & $30 \mathrm{~min}$ & $40 \mathrm{~min}$ \\
\hline Ship A & & & & \\
\hline Cabin No.1 & $6.5 \%$ & $5.7 \%$ & $5.5 \%$ & $4.5 \%$ \\
\hline Cabin No.2 & $5.1 \%$ & $5.6 \%$ & $4.9 \%$ & $4.7 \%$ \\
\hline Cabin No.3 & $6.3 \%$ & $6.0 \%$ & $5.2 \%$ & $4.3 \%$ \\
\hline Cabin No.4 & $6.5 \%$ & $5.4 \%$ & $5.1 \%$ & $4.3 \%$ \\
\hline Cabin No.5 & $5.7 \%$ & $5.3 \%$ & $5.0 \%$ & $4.6 \%$ \\
\hline Ship B & & & & \\
\hline Cabin No.1 & $5.1 \%$ & $5.2 \%$ & $4.8 \%$ & $4.5 \%$ \\
\hline Cabin No.2 & $5.6 \%$ & $6.1 \%$ & $4.5 \%$ & $4.0 \%$ \\
\hline Cabin No.3 & $6.2 \%$ & $5.4 \%$ & $4.6 \%$ & $4.1 \%$ \\
\hline Cabin No.4 & $5.8 \%$ & $5.1 \%$ & $5.3 \%$ & $4.4 \%$ \\
\hline
\end{tabular}

\section{CONCLUSION}

A large number of continuous experiment against automatic ship loader of bulk terminal proved that the anti-collision method described in this paper can detect the original outline shape of the cabin and cargo precisely by laser radar array, exclude the block of shovel and cargo being thrown with compensation algorithm for helping to identify the position and height of the cargo more accurately. The method can serve in most automated ship loader or manual one and maintain a

3. Mi Chao, Zhang Zhiwei, He Xin, Huang Youfang, Mi Weijian. "Two-Stage Classification Approach for Human Detection in Camera Video Bulk Ports," Polish Maritime Research, Vol. 22, No. 86, pp. 163-170 (2015).

4. Song, Gyeeui , "A Study on Improvement of Handling Dirty Bulk Cargo in Busan Port," JOURNAL OF KOREA PORT ECONOMIC ASSOCIATION, Vol. 26, No. 3, pp.114-129 (2010)

5. Froyland, Gary; Koch, Thorsten; Megow, Nicole; "Optimizing the landside operation of a container terminal,” OR SPECTRUM, Vol. 30, No. 1 pp. 53-75 (2008)

6. Mi Chao, Zhang Zhiwei, Huang Youfang, Shen Yang, "A FAST AUTOMATED VISION SYSTEM FOR CONTAINER CORNER CASTING RECOGNITION," Journal of Marine Science and Technology-Taiwan, Vol. 24, No. 1, pp. 54-60 (2016)

7. Choe, Ri; Kim, Jeongmin; Ryu, Kwang Rye, "Online preference learning for adaptive dispatching of AGVs in an automated container terminal," APPLIED SOFT COMPUTING, Vol. 38, pp. 647-660 (2016)

8. Mi Chao, Huang Youfang, Liu Haiwei, Shen Yang, Mi Weijian. "Study on Target Detection \& Recognition Using Laser 3D Vision System for Automatic Ship Loader," Sensor \& Transducers, Vol. 158, No.11, pp. 436-442 (2013).

9. Zupancic, Nina; Horvat, Aleksander; Skobe, Simona, "Environmental impact of dusting from the Koper port bulk cargo terminal on the agricultural soils," ACTA GEOGRAPHICA SLOVENICA-GEOGRAFSKI ZBORNIK, Vol. 55, No.1, pp. 140-158 (2015)

10. Mi Chao, He Xin, Liu Haiwei, Huang Youfang, Mi Weijian. "Research on a Fast Human-Detection Algorithm for Unmanned Surveillance Area in Bulk Ports," Mathematical Problems in Engineering, (2014). 
11. Liu Pengfei, Lin Bin, Zhang Xiaofeng, "Safety and Stability Analysis of the Ship loader during Shipping," ADVANCES IN MATERIALS MANUFACTURING SCIENCE AND TECHNOLOGY XV, Vol. 777, pp. 189-193 (2014)

12. Mi Chao, Shen Yang, Mi Weijian, Huang Youfang. "Ship Identification Algorithm Based on 3D Point Cloud for Automated Ship Loaders," Journal of Coastal Research, Vol. 73, pp. 28-34 (2015).

13. Gang Zhou. "Automobile Anti-collision Millimeter-Wave Radar Signal Processing," Intelligent Human-Machine Systems and Cybernetics (IHMSC), Vol. 2, pp. 484-486 (2015)

14. Frederic Maire. "Vision based anti-collision system for rail track maintenance vehicles," Advanced Video and Signal Based Surveillance, pp. 170-175 (2007)

15. Mi Chao, Liu Haiwei, Huang Youfang, Mi Weijian, Shen Yang, Fatigue alarm systems for port machine operators, ASIA LIFE SCIENCES, Vol. 25, No.1, pp. 31-41 (2016)

16. Mi Chao, Liu Haiwei, Zhao Ning, Shen Yang. "A ship cargo hold inspection approach using laser vision systems," Telkomnika, pp. 330-337 (2013).

17. Rodriguez-Cuenca Borja, Garcia-Cortes Silverio, Ordonez Celestino, "An approach to detect and delineate street curbs from MLS 3D point cloud data," AUTOMATION IN CONSTRUCTION, Vol. 51, pp. 103-112 (2015)

18. Diaz-Vilarino Lucia, Conde Borja, Lagueela Susana, "Automatic Detection and Segmentation of Columns in As-Built Buildings from Point Clouds," REMOTE SENSING, Vol.7, No. 11, pp. 15651-15667 (2015)

19. Tsz Ching Ng, Gu Xianfeng, Lui Lok Ming, “Computing Extremal Teichmuller Map of Multiply-Connected Domains Via Beltrami Holomorphic Flow," JOURNAL OF SCIENTIFIC COMPUTING, Vol. 60, No. 2, pp. 249 275 (2014)

20. Wei Xiangzhi, Joneja Ajay, "On computing the shortest path in a multiply-connected domain having curved boundaries," COMPUTER-AIDED DESIGN, Vol. 48, pp. 39-41 (2014)

21. Paille Gilles-Philippe, Poulin Pierre, Levy Bruno, "Fitting Polynomial Volumes to Surface Meshes with Voronoi Squared Distance Minimization," COMPUTER GRAPHICS FORUM, Vol. 32, No. 5, pp. 103-112 (2013)

22. Luo Ding, Kuang Cuifang, Liu Xu, "Fiber-based chromatic confocal microscope with Gaussian fitting method," OPTICS AND LASER TECHNOLOGY, Vol. 44, No. 4, pp. 788-793 (2012)
23. Chan-Hon-Tong Adrien, Achard Catherine, Lucat Laurent, "Simultaneous segmentation and classification of human actions in video streams using deeply optimized Hough transform," PATTERN RECOGNITION, Vol. 47, No. 12, pp. 3807-3818 (2014)

24. Jiang, Haibo; Tang, Kai; He, Xinlin, “Experimental Studies on Reduction of Evaporation from Plain Reservoirs in Drought Areas by Benzene Board Covering Technology," JOURNAL OF COASTAL RESEARCH, Vol. 73, pp. 177$182(2015)$

25. Lazarowska, Agnieszka, "Swarm Intelligence Approach to Safe Ship Control”, POLISH MARITIME RESEARCH. Vol. 22, No. 4, pp. 34-40 (2015)

\section{CONTACT WITH THE AUTHOR}

\author{
SHEN Yang \\ yangshen@shmtu.edu.cn \\ Higher Technical College \\ Shanghai Maritime University \\ Shanghai \\ CHINA
}

